\title{
Molecular characterization of influenza virus in intestines and its effect on intestinal microbiota
}

Hebah A. Al Khatib ${ }^{1}$, Muna Al Maslamani ${ }^{2}$, Peter Coyle ${ }^{2}$, Sameer Pathan ${ }^{2}$, Asmaa Al Thani ${ }^{3}$, Hadi M. Yassine ${ }^{3}$

${ }^{1}$ College of Science and Engineering, Hamad Ben Khalifah University, ${ }^{2}$ Hamad Medical Corporation, ${ }^{3}$ Biomedical Research Center, Qatar University

\section{BACKGROUND}

Influenza predominantly causes respiratory illness; with gastrointestinal symptoms commonly reported in about $30 \%$ of patients. Influenza virus RNA has been also detected in stools of patients infected with pandemic and seasonal influenza, however, the role and the clinical significance of intestinal infection has not been clearly demonstrated.

Here we aim to (i) study the impact of influenza infection on intestinal microbiota; and (ii) characterize influenza viruses isolated from fecal samples of infected patients and their potential role in virus transmission.
1 Sample collection
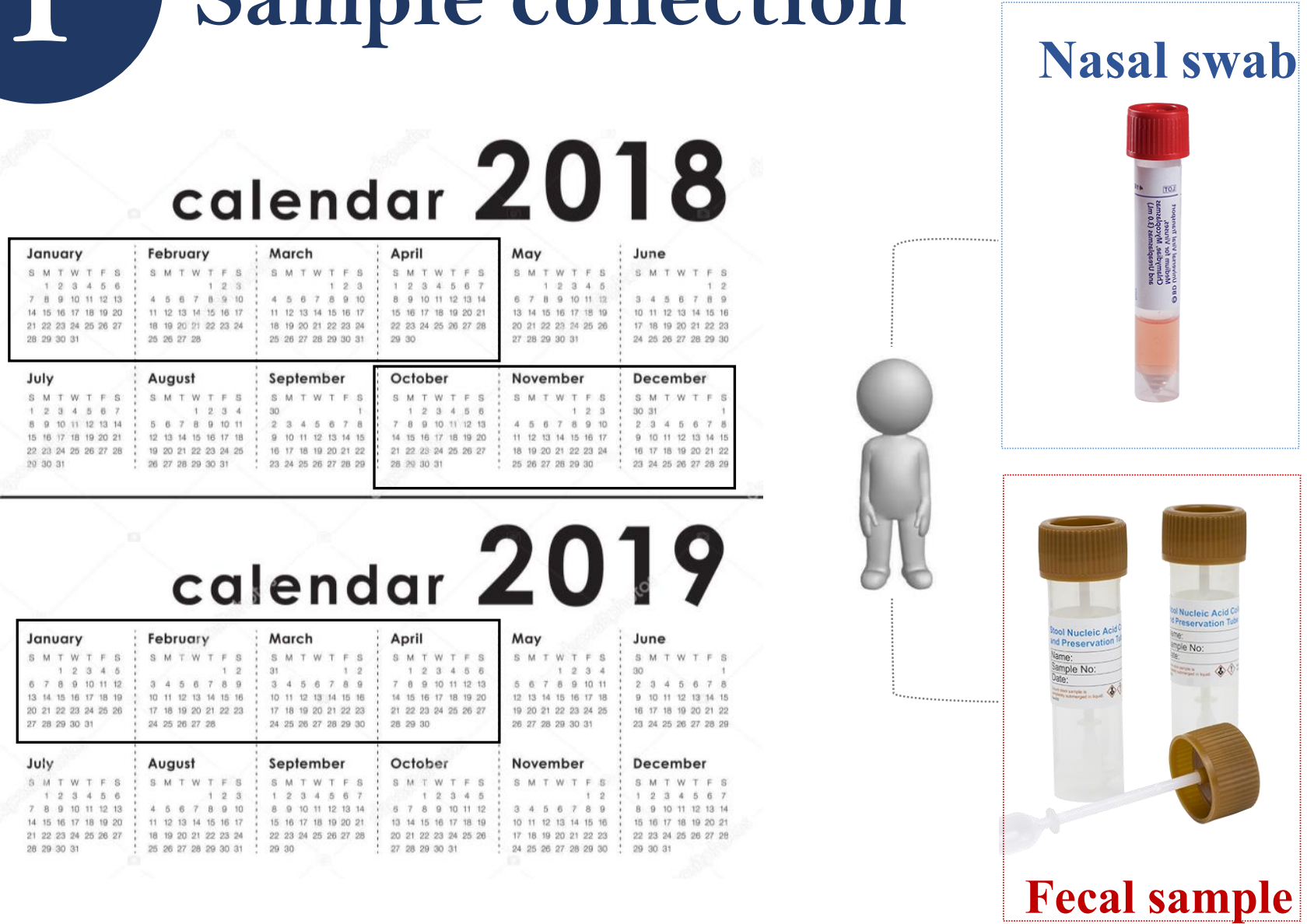
2
METHODS
Influenza Detection

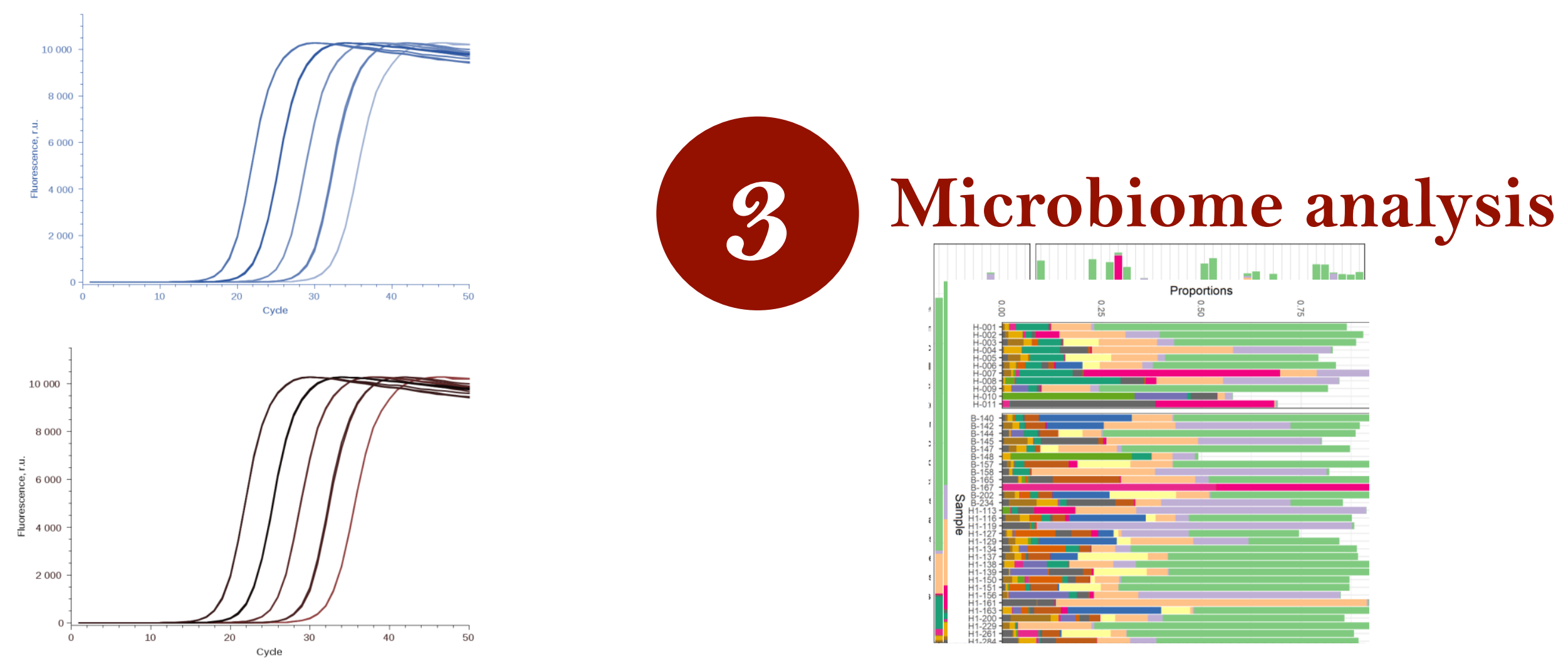
5
4. Cell culture

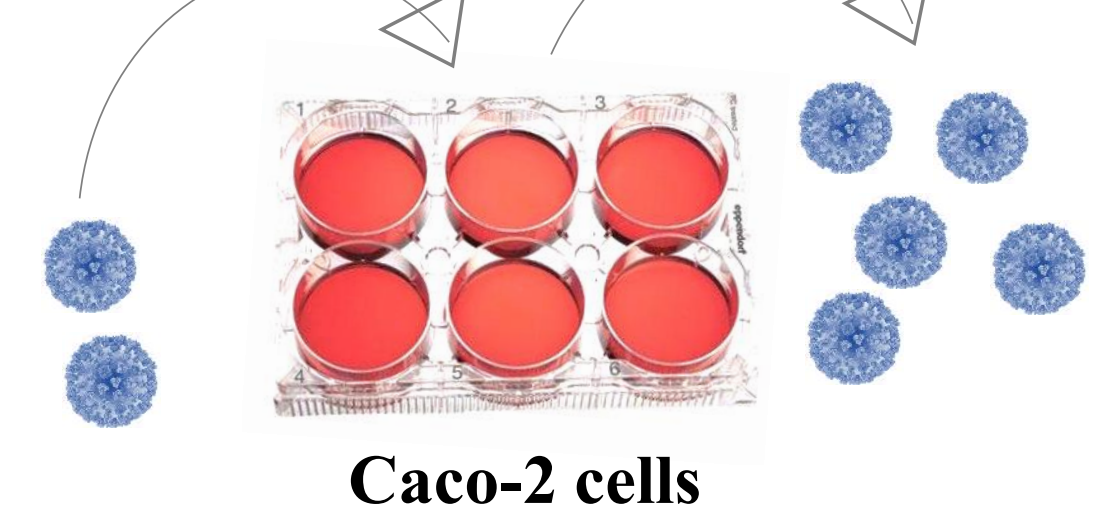
NGS

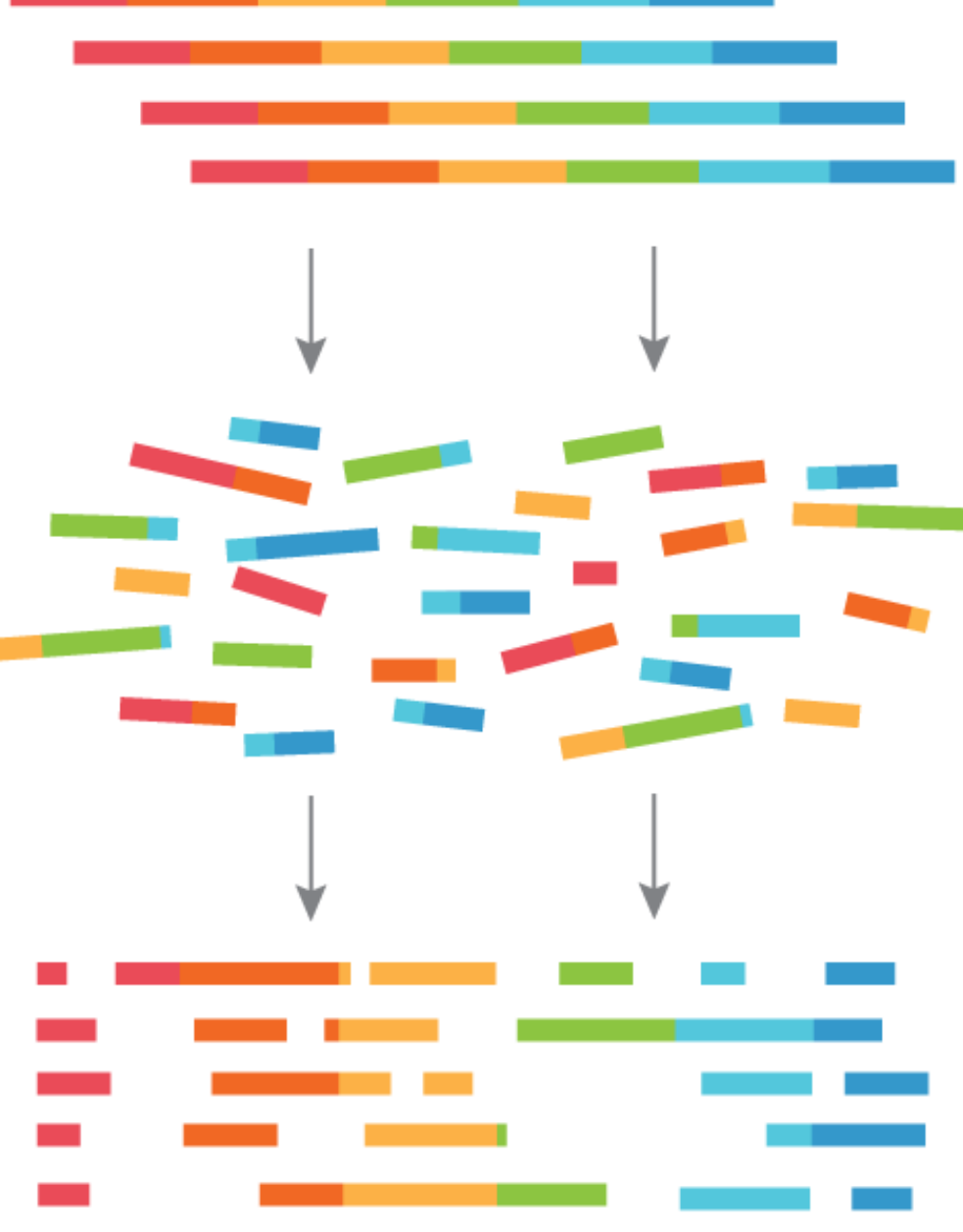

\section{RESULTS}

\section{MICROBIOME}

1. Changes in gut microbiota composition is directly related to influenza type. A total of 10 OTUs were found to be differentially abundant among influenza $\mathrm{A}-$ and $\mathrm{B}$ infected patients. Of these, the relative abundance of 6 OTUs were significantly higher in influenza B patients while 4 OTUs were more abundant in influenza A patients.

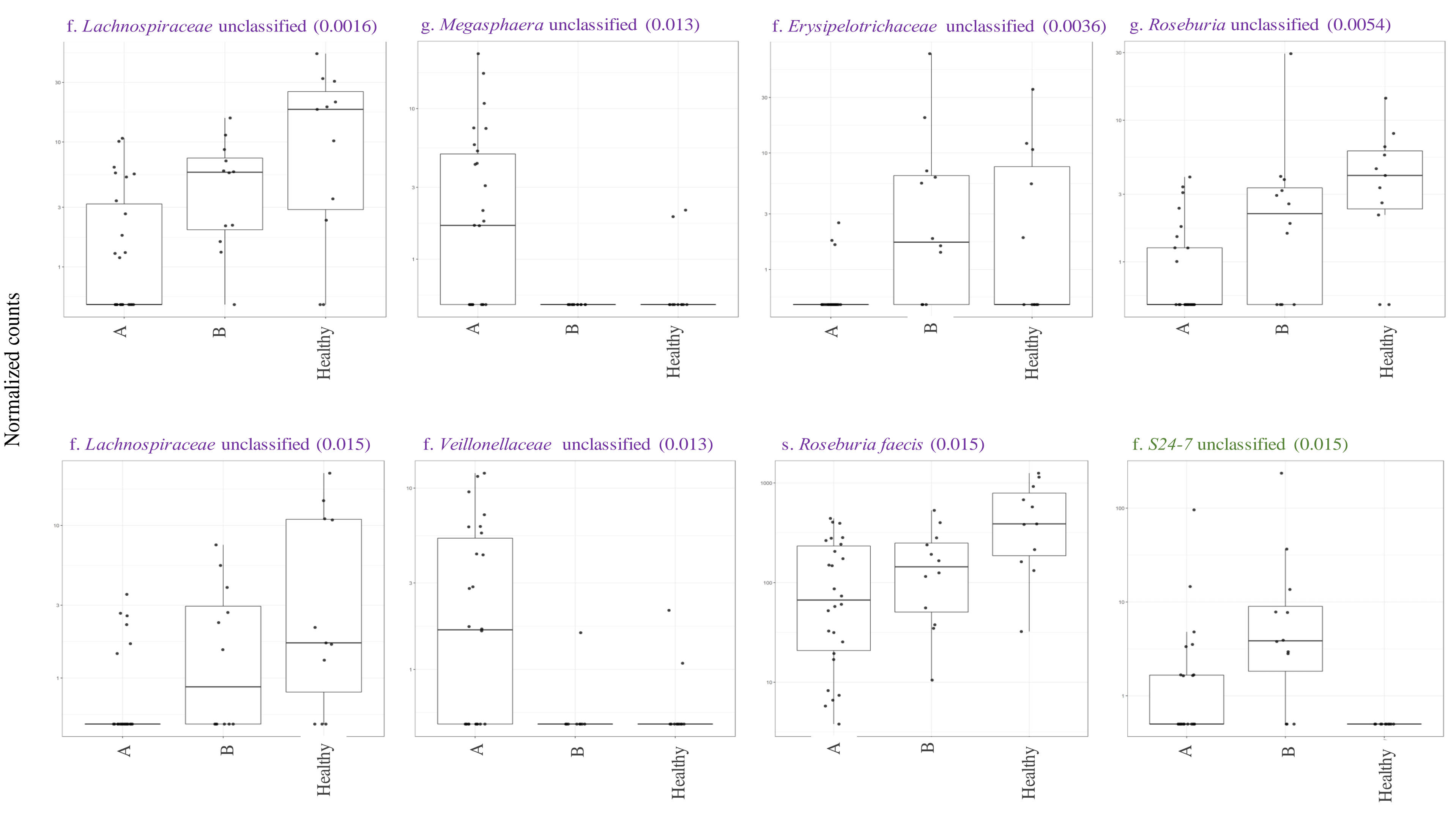

OTUs are colored based on phyla: purple for Firmicutes and green for Bacteroidetes. The Padj value for each OUT is indicated between brackets.

2. Comparison of intestinal bacteria diversity and composition in influenza-infected patients who were shedding virus in their stools (S) and those who were not (NS).

(A) Microbial profiles (at genus level) of shedders and non-shedders among $\mathrm{H} 1 \mathrm{~N} 1$ and $\mathrm{H} 3 \mathrm{~N} 2$ infected patients. (B) Alpha diversity of intestinal microbiota was significantly lower among shedders compared to non-shedders of $\mathrm{H} 1 \mathrm{~N} 1$ and $\mathrm{H} 3 \mathrm{~N} 2$ infected patients.

A

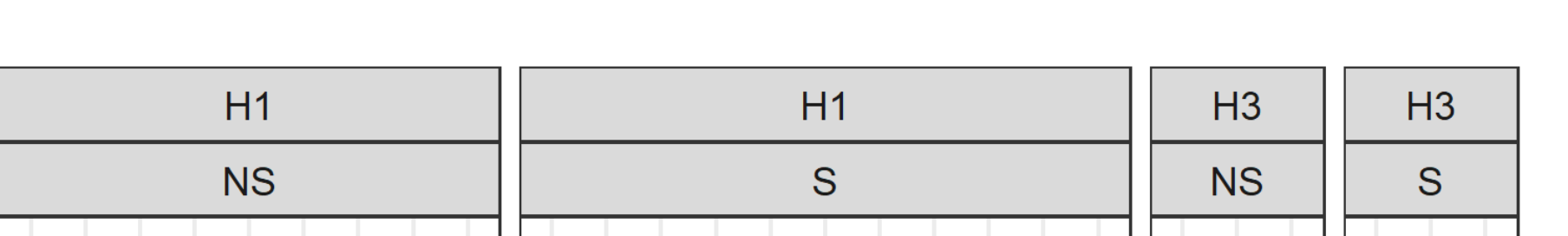

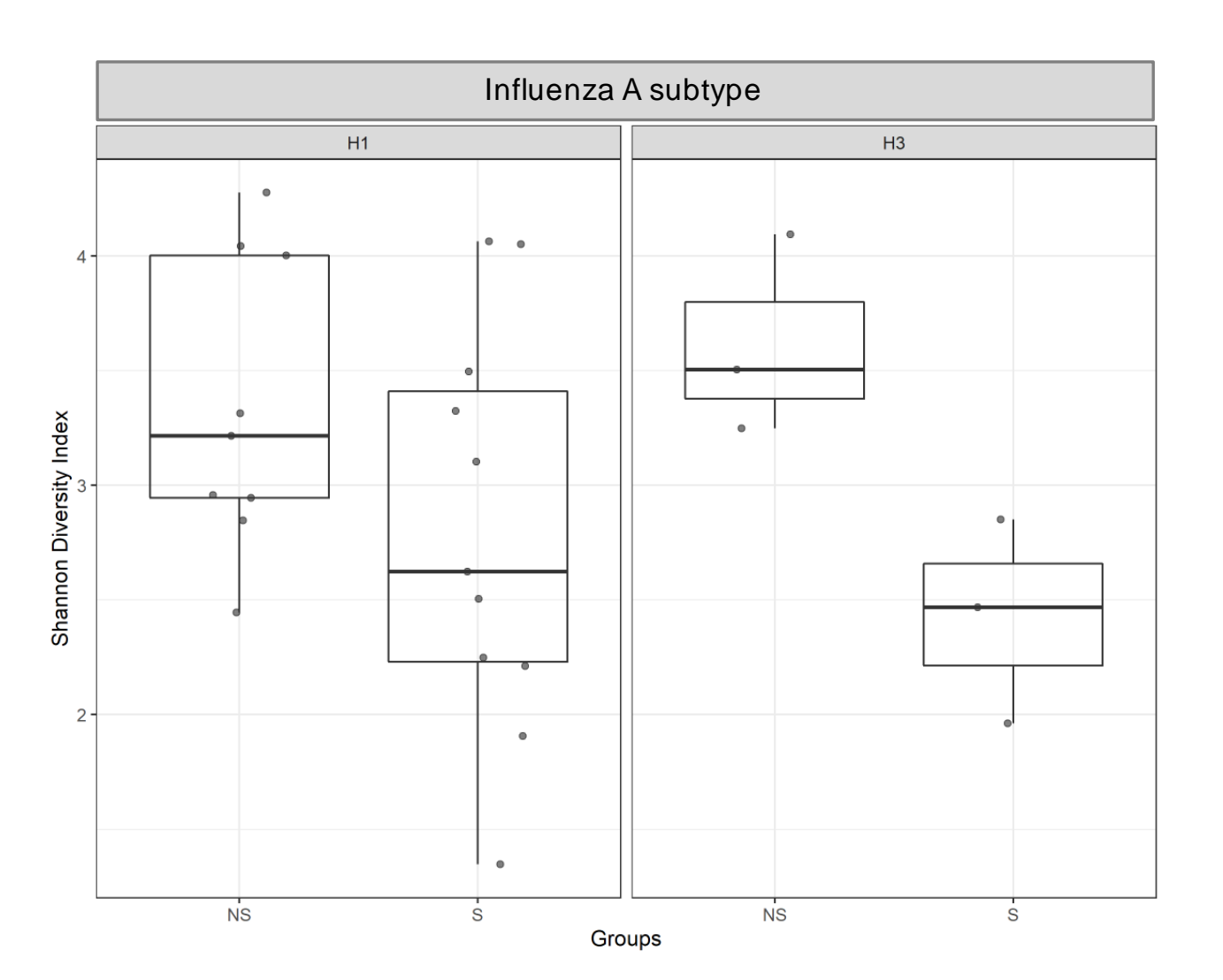

\section{CONCLUSION}
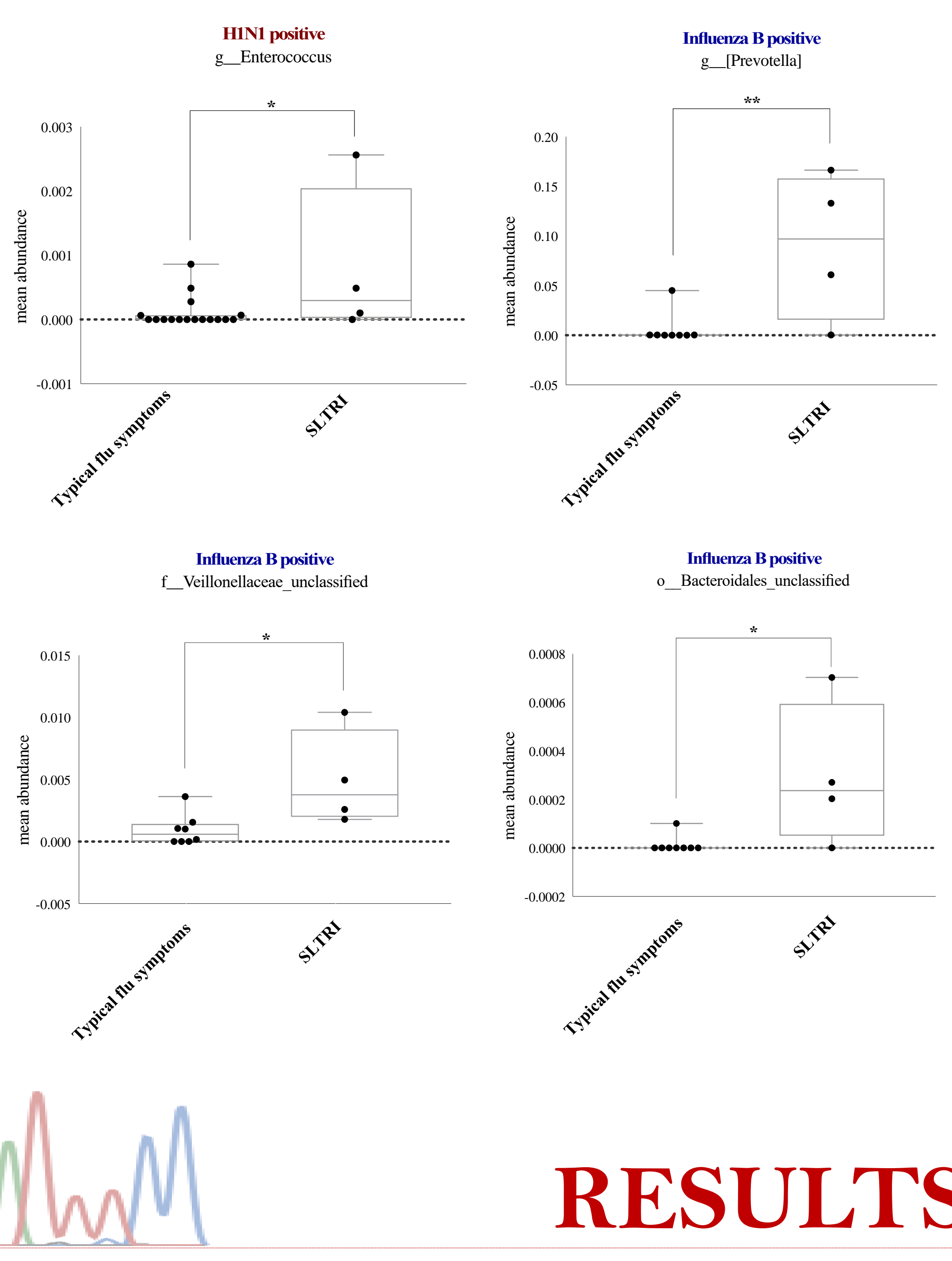

RESULTS SEQUENCING

1. Mutations detected in HA protein of influenza viruses isolated from intestinal origin

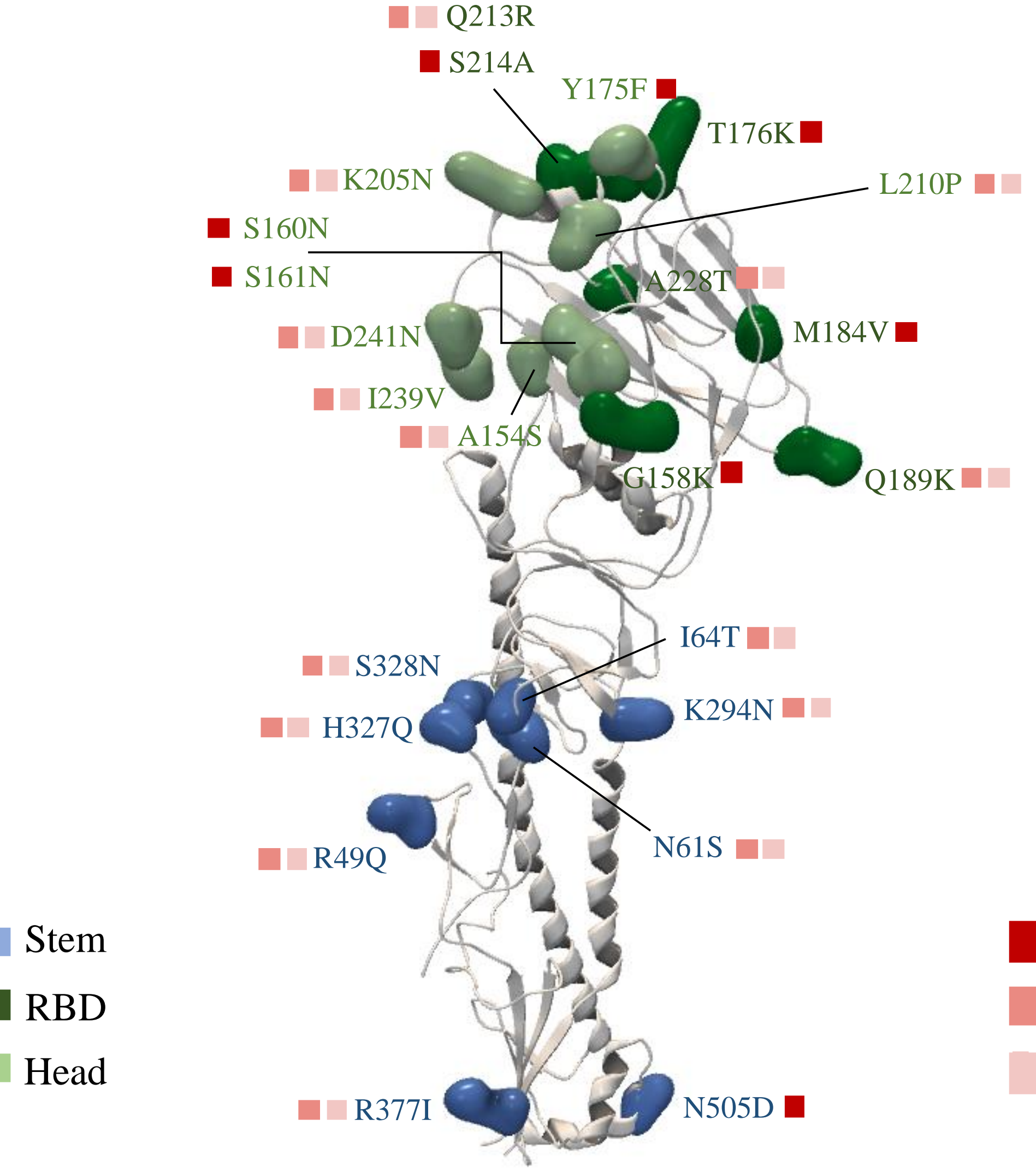

Increased fitness least fitnes

Sequencing and microbiome analysis suggest that influenza viruses can affect intestinal environment either by direct intestinal infection or indirectly by modulating intestinal microbiota.

changes in gut microbiota composition in influenza-infected patients are significantly associated with:

$>$ influenza virus type

$>$ the presence of viral RNA in intestines of infected patients 